\title{
A first report of Thelazia callipaeda infection in Phortica okadai and wildlife in national nature reserves in China
}

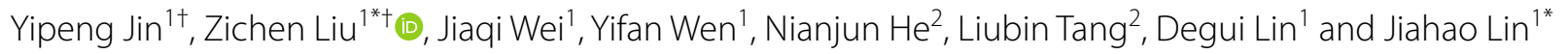

\begin{abstract}
Background: Thelazia callipaeda is a zoonotic parasitic nematode of the family Thelaziidae, with Phortica okadai as its intermediate host and only confirmed vector in China. China has the largest number of human cases of thelaziosis in the world. It is generally believed that infected domestic animals (dogs and cats) are the most important reservoir hosts of T. callipaeda, and thus pose a direct threat to humans. At present, there is little research or attention focused on the role of wild life in the transmission cycle of thelaziosis in nature reserves.

Methods: We selected locations in four national nature reserves across China to monitor P. okadai and wildlife. We used a fly-trap method to monitor P. okadai density. Morphological analysis of the parasites collected from the conjunctival sac of the infected wildlife was undertaken as the first step in species identification, and polymerase chain reaction (PCR) was used for species confirmation.
\end{abstract}

Results: In 2019, the density of P. okadai in Foping National Nature Reserve in China increased sharply, and infected P. okadai were newly found in the reserve. Giant panda, wild boar, leopard cat, and black bear were found to be newly infected with T. callipaeda (one individual of each species). A total of four worms were collected, one from each species of wild life. The four worms were identified as T. callipaeda by their morphological characteristics; species identification was confirmed by PCR amplification.

Conclusions: To the best of our knowledge, this is the first report of T. callipaeda infection in P. okadai as well as in a variety of wildlife, including giant panda, in nature reserves in China. These results indicate that there is a transmission cycle of T. callipaeda among wildlife in these nature reserves. The increasing number of case reports of thelaziosis in wild life suggest a likely risk of T. callipaeda infection for the inhabitants of villages situated around nature reserves.

Keywords: Thelazia callipaeda, Phortica okadai, Wildlife, Vector-borne zoonosis, Nature reserves, China

\section{Background}

Thelazia callipaeda is a zoonotic parasitic nematode of the family Thelaziidae, with Phortica okadai (Diptera, Drosophilidae, Steganinae) as its intermediate host

\footnotetext{
*Correspondence: Izc94@126.com; jiahao_lin@cau.edu.cn

${ }^{\dagger}$ Yipeng Jin and Zichen Liu contributed equally to this work

${ }^{1}$ College of Veterinary Medicine, People's Republic of China Agricultural

University, No. 2 Yuanmingyuan West Road, Haidian District,

Beijing 100193, People's Republic of China

Full list of author information is available at the end of the article
}

and only confirmed vector in China [1-5]. The definitive host of $T$. callipaeda varies widely, and includes wildlife, domestic animals and humans [6]. Dogs are its most important reservoir host. T. callipaeda parasitizes the conjunctival sac and lacrimal duct of mammals. Its reproductive mode is ovoviparous [7]. Infective larvae of $T$. callipaeda escape from $P$. okadai's proboscis when the insect licks the eye of a mammal and invade the conjunctival sac [4]. The damage caused by parasitic T. callipaeda to the eyes of animals can differ in 
severity. The host's eye can be damaged by the friction of the sharp folds of the surface of the worm and adsorption of the mouth sac by the eye tissue. In addition, adult worm secretions and excreta stimulate the tissues of the eye. During clinical examination, some infected dogs showed foreign body sensation, increased ocular secretion, eyelid edema, conjunctival congestion, inflammation or formation of small ulcers, turbidity of the aqueous humour, and increased intraocular pressure $[7,8]$.

In recent years, many countries have reported cases of humans infected with T. callipaeda, and there have also been reports of dogs and wild host animals infected with T. callipaeda in Italy, Germany, Serbia and other European countries [9-11]. From 1917 to 2019, a total of 643 human cases of thelaziosis were recorded in China $[12,13]$. China has the largest number of human cases of thelaziosis in the world, and saw a significant increase in cases between 1970 and 1999. Although the number of human cases of thelaziosis has decreased in the past 20 years, it is starting to increase again $[12,13]$. An increase in the number of $P$. okadai coupled with the proliferation of domestic animals (dogs and cats) has led to increased vigilance towards T. callipaeda infection.

The T. callipaeda infection rate of villagers is higher than that of urban dwellers [13] because the village environment is more suitable for the survival of $P$. okadai. We performed ocular examinations of domestic animals (dogs) from 2016 to 2019 in the villages around national nature reserves and found that, in 2019, the prevalence rate of thelaziosis was as high as $84.62 \%$ (88/104), which was higher than that in 2016 (38.05\%, 43/113), 2017 (53.92\%, 55/102) and 2018 (56.25\%, 63/112).

Most nature reserves in China are surrounded by villages, and the number of wild animals in the reserves far exceeds the number of domestic animals in the villages. Thus, if the wildlife spread T. callipaeda on a large scale, they could pose a threat to the inhabitants of nearby villages and their domestic animals.

We selected four national nature reserves across China to monitor the prevalence of T. callipaeda in $P$. okadai and wildlife between 2016 and 2019. The four monitoring locations were located in the home range of the giant panda, one of the most complex topographical regions in the world, with clear vertical zoning of climate, and home to more than 8000 confirmed species of wildlife and plants. In the villages around these reserves, some villagers own domestic animals. These locations were selected for this study as the home ranges of the wildlife of the reserves and the domestic animals (dogs and cats) of the surrounding villages overlap within them [14]. The reserves were also selected because they are close to densely populated cities.

\section{Methods \\ Monitoring locations for $P$. okadai and wildlife}

We selected locations in the following four national nature reserves, which are situated across China, for the monitoring of $P$. okadai and wildlife between 2016 and 2019: Foping National Nature Reserve, Shaanxi (FNNR; $\left.33^{\circ} 38^{\prime} 43^{\prime \prime} \mathrm{N}, 107^{\circ} 47^{\prime} 38^{\prime \prime} \mathrm{E}\right)$; Tangjiahe National Nature Reserve, Sichuan $\left(32^{\circ} 34^{\prime} 44^{\prime \prime N}, 104^{\circ} 45^{\prime} 43^{\prime \prime E}\right) ;$ Wolong National Nature Reserve, Sichuan (WNNR; $31^{\circ} 02^{\prime} 20^{\prime \prime N}$, $\left.103^{\circ} 11^{\prime} 52^{\prime \prime} \mathrm{E}\right)$; and Fengtongzhai National Nature Reserve, Sichuan $\left(30^{\circ} 22^{\prime} 05^{\prime \prime N}, 102^{\circ} 48^{\prime} 52^{\prime \prime E}\right)$ (Fig. 1).

\section{Monitoring P. okadai density and morphological identification}

In accordance with the surveillance methods for vector density-fly promulgated by the National Health Commission of the People's Republic of China [15], we used a fly-trap method to monitor $P$. okadai density. A total of 200 traps were placed at each monitoring location, of which 100 were distributed in the home ranges of wildlife and the other 100 in the surrounding villages. The traps were baited with prepared fruit mash. Monitoring was done from April to October. The fly traps were placed at the monitoring locations at 9 a.m. in the middle of each month, and were retrieved at 9 a.m. on the following day. Because many kinds of fly were caught in the traps, P. okadai were first identified according to morphological characteristics. They were then removed from the traps for further morphological analysis under a microscope. The density of $P$. okadai (number per trap per $24 \mathrm{~h}$ ) was calculated and the infection rate of T. callipaeda determined by dissection [15].

\section{Sample collection}

In 2019, four cases of ocular worm infection were identified in FNNR, one in giant panda (Additional file 1: Figure S1), one in wild boar, one in leopard cat and one in black bear. Infection was determined by ophthalmic examination of the animals under anesthesia in FNNR; anesthetic was given by injection. A total of four worms were collected, one from each animal.

\section{Parasite collection and treatment}

The worms were removed from the conjunctival sac and placed in a sampling tube containing $70 \%$ ethanol. Morphological analysis of the parasites was the first step in species identification; PCR was used for species confirmation. 


\section{Morphological analysis}

The parasites collected from the eyes of each animal were examined under a light microscope combined with a camera and identified on the basis of their morphology by using taxonomic keys.

\section{Sequence and phylogenetic analysis}

We extracted genomic DNA of each worm from the conjunctival sac of the giant panda, wild boar, leopard cat, and black bear with the HiPure Tissue \& Blood DNA Kit (MAGEN, China). A partial sequence of the mitochondrial cytochrome c oxidase subunit 1 gene (cox1; 689 base pairs) was amplified by PCR. Amplicons were purified by a HiPure Gel Pure Micro Kit (MAGEN, China) and sequenced in an ABI3730XL with the BigDyeTr v3.1
Cycle Seq Kit (Applied Biosystems, USA). Amplicon sequences were determined in both directions (GenBank accession nos. MN719908, MN719912, MN719913 and MN719914) and genetic analyses performed using available sequences of related nematodes from GenBank and the Global Initiative on Sharing all Influenza Data (GISAID) database (https://www.gisaid.org). The phylogenetic tree (Fig. 2) was constructed by MEGA version 6 (https://www.megasoftware.net) using the neighbor-joining method with 1000 bootstrap replicates $[9,10,16,17]$.

\section{Results}

Examination of the worms under a microscope (Additional file 1: Figure S2) showed characteristic morphological features of P. okadai $[2,5,18]$; the genitalia were not examined.

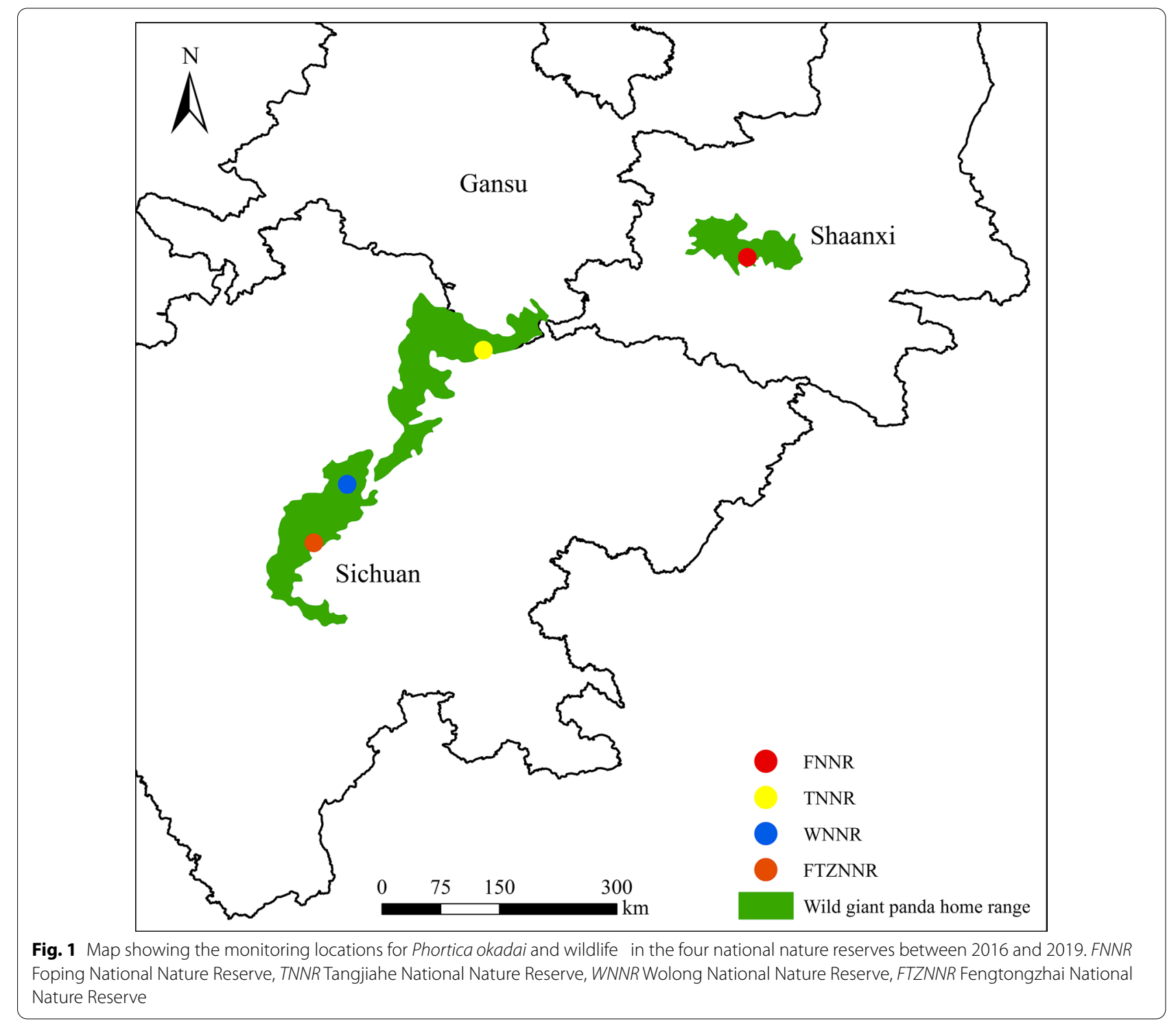


Before 2019 (in 2016, 2017 and 2018), there were fewer $P$. okadai in the wildlife home ranges than in the surrounding villages; no infected $P$. okada $i$ were found in the wildlife home ranges during this period (Tables 1 , $2,3)$. A high number of infected $P$. okadai were found in the surrounding villages during this time (Tables 1 , 2 , 3). In 2019, the density of P. okadai increased sharply in FNNR, and infected $P$. okadai were newly found in the home ranges of the reserve's wildlife (Table 4). The distribution density of $P$. okadai was highest in July and August, and the highest numbers of $P$. okadai infected with larvae of $T$. callipaeda were also found in these months (Fig. 3a).

Morphological observation of the collected parasites showed that they had a cylindrical shape with thin ends, and were milky white in color and slightly transparent. All of the worms were female. The mean body length and width of the females were $13.7 \mathrm{~mm}$ and $0.36 \mathrm{~mm}$, respectively. The buccal capsule, pharynx, and esophagus of the anterior segment of the worm body, its serrated, wrinkled surface and the caudal end of the worm were visible under the optical microscope (Fig. 3b, c). Larvae and oval shaped eggs were found in the mid-section of the uterus (Fig. 3d). The worms were identified as $T$. callipaeda from their morphological characteristics.

The $2 \%$ agarose gel electrophoresis of the PCR amplified products of each sample showed a DNA band of about 689 base pairs in length, which was consistent with the expected size of the T. callipaeda cox 1 sequence (Fig. 4). There were no non-specific bands. The alignment and phylogenetic analyses of the neighbor-joining method showed that the $T$. callipaeda cox 1 sequences of giant panda, wild boar and leopard cat (GenBank accession nos. MN719908, MN719912 and MN719913) clustered with those of T. callipaeda in dogs, cats, and humans from China, Japan and Korea. The sequences from the black bear (GenBank accession nos. MN719914) were closely related to those from European animals, as they were clustered together. All the cox 1 sequences of $T$. callipaeda clustered into one large branch, while the cox 1 sequences of Onchocerca lupi from the USA formed an outgroup (Fig. 2).

We can confirm from morphological observations and $\operatorname{cox} 1$ detection that the ocular worms, of which there were four in total, collected from a giant panda (Additional file 1: Figure S1), wild boar, leopard cat and black bear (one individual of each species) were T. callipaeda.

\section{Discussion}

It is generally believed that domestic animals are the most important reservoir hosts of T. callipaeda, and thus pose a direct threat to humans $[6,8,11,19-21]$. However, in the present study, we identified cases of thelaziosis in giant panda, wild boar, leopard cat, and black bear in 2019. In the same year, a large number of the intermediate host, $P$. okadai, were also found to be infected in the home ranges of wildlife in FNNR. Thus, we believe that thelaziosis has already spread in this nature reserve. The outbreak of T. callipaeda infections in P. okadai within the home ranges of wildlife in FNNR shows that the transmission cycle of this parasite has been established there.

Summer is the most active season for the intermediate host of T. callipaeda [22], and is also the season in which numerous wildlife species migrate in nature reserves [14, 23-25]. Newborn larvae of T. callipaeda are produced directly in the conjunctival sac of infected wildlife. When the intermediate host, $P$. okadai, licks the secretions of an infected animals's eye, it sucks the newborn larvae into its body. After ecdysis of the larvae, which occurs twice, they develop into infective larvae, at which point they enter the head and proboscis of the $P$. okadai. When the P. okadai then licks the eye of another wildlife host, the infective larvae of T. callipaeda escape from the proboscis and invade the new host's conjunctival sac. It usually takes $15-20$ days for a larva to develop into an adult [7]. Female T. callipaeda can continue to produce larvae until they are about 35 days old; these larvae are then licked from the host's eye by the intermediate host, P. okadai, and thus the cycle continues. Thus, P. okadai infected with T. callipaeda pose a threat to both domestic animals and inhabitants of the villages surrounding the nature reserves.

Once thelaziosis is widespread among the wildlife of the reserves, villagers in the surrounding villages will inevitably be susceptible to infection due to their frequent activities near the home ranges of the wildlife. If thelaziosis is not controlled, the infected wildlife will gradually form a bank of reservoir hosts among the wildlife of the reserves. As winter approaches, wildlife migrate to the villages, which leads to interactions between wildlife and domestic animals [14, 23-25]. These interactions create an opportunity for the transmission of T. callipaeda between wildlife and domestic animals by $P$. okadai.

FNNR had the highest density of P. okadai compared to the other studied biotopes, due to its geography and climate [22]. FNNR is located in Foping county, northeast Hanzhong city, Shaanxi Province, within the northern sub-tropical climate zone. The Qinling mountains to the north of FNNR and Daba mountains to the south, which have an altitude range of 980-2904 $\mathrm{m}$, form two natural barriers. As humid air does not spread easily northwards, the climate of FNNR is mild and humid. This climate is suitable for the vector of $T$. callipaeda, P. okadai. P. okadai was not found in the 


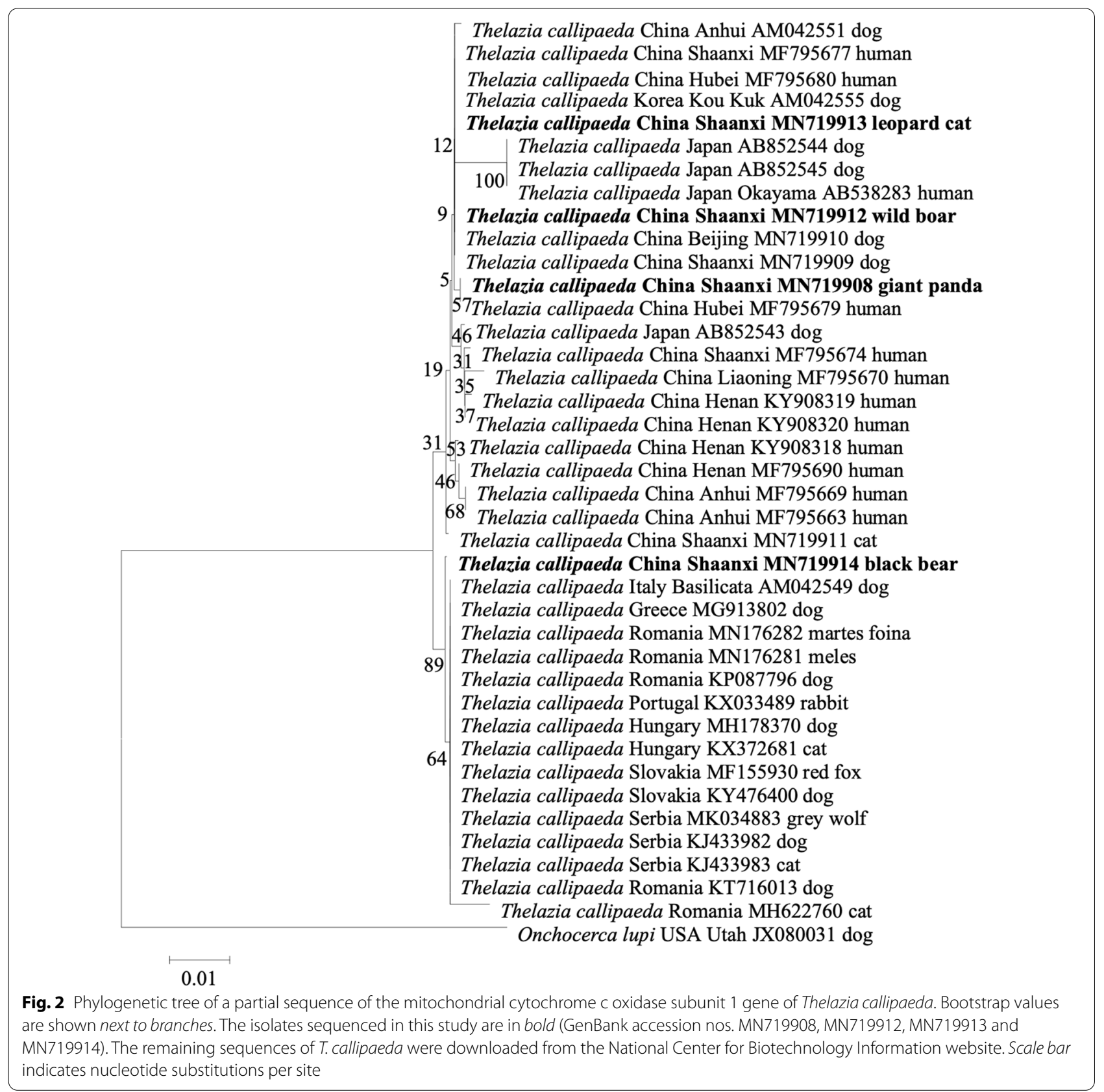

WNNR monitoring points. The reason for this is that most of the mountain peaks there are over $4000 \mathrm{~m}$ in altitude, and the average annual temperature is $8.5 \pm 0.5{ }^{\circ} \mathrm{C}$, which seems to be too low for the survival of P. okadai.

In this study, only four T. callipaeda were collected in total, i.e. an individual worm from four species of wildlife. The animals had to be anesthetized for parasite collection, and sampling was extremely problematic due to the large home ranges and irregular movements of the animals. Therefore, to better elucidate the actual number of animals and species of wildlife infected, we need to continue to sample and screen.

We selected a partial sequence of the mitochondrial cyt1 gene as a genetic marker for the phylogenetic analysis of the parasites collected from the eyes of the infected animals. Because of its small molecular weight and strongly conserved structure, many researchers consider that cyt 1 can be used as a specific genetic marker for some nematodes. As early as 2005, Dominico Otranto and coworkers successfully analyzed the genetic variability of T. callipaeda from Europe and Asia by sequencing 


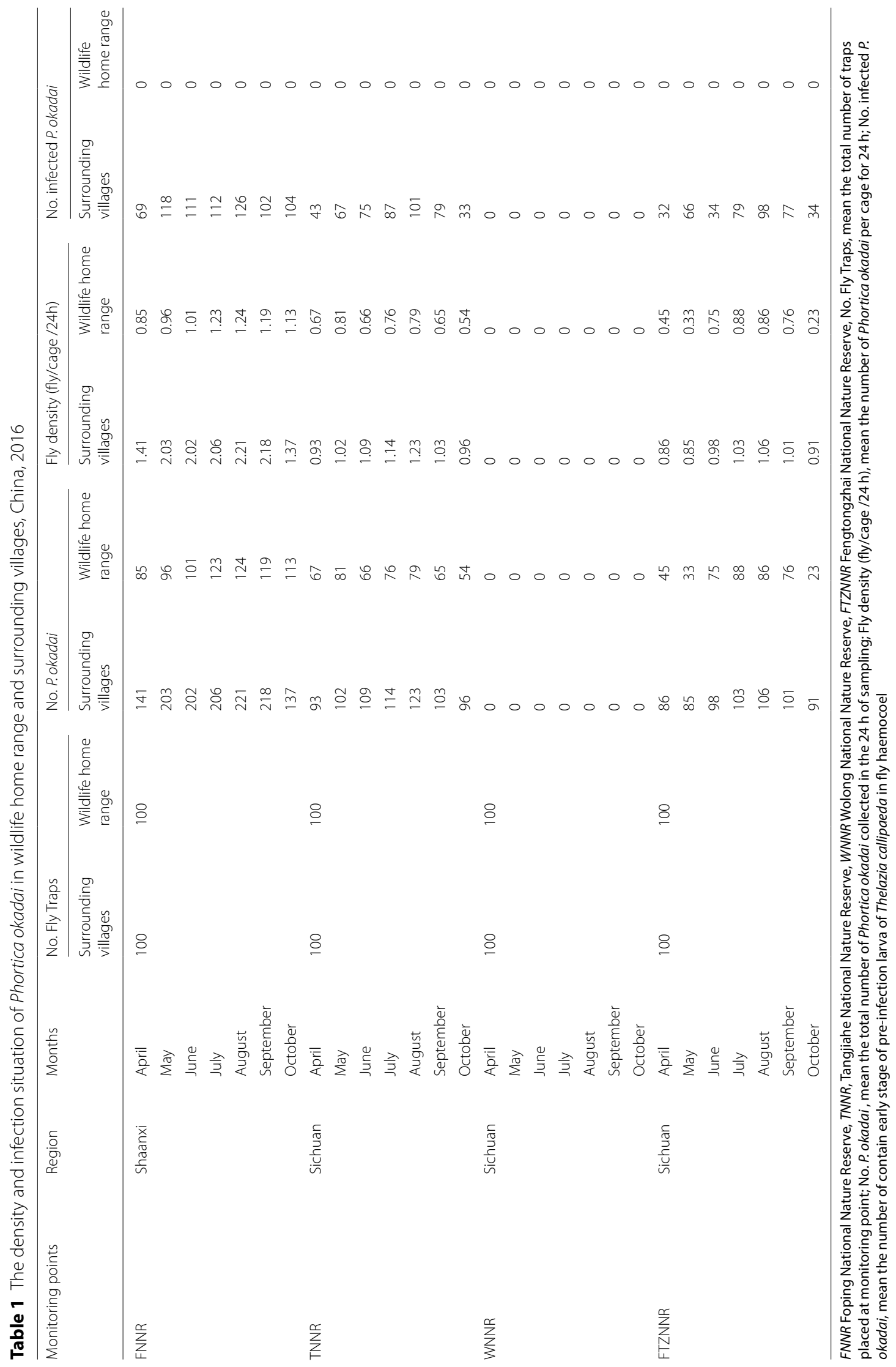




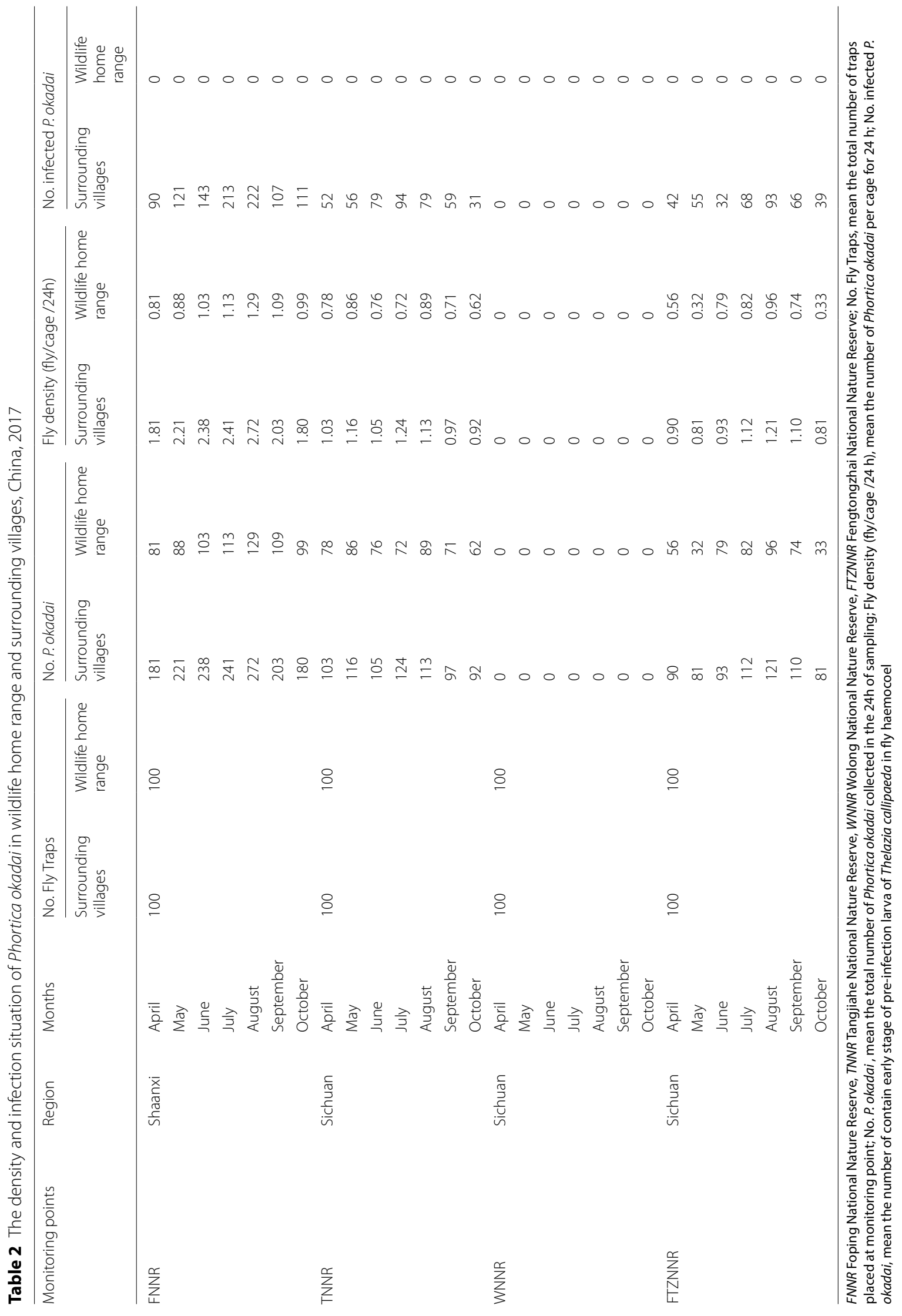




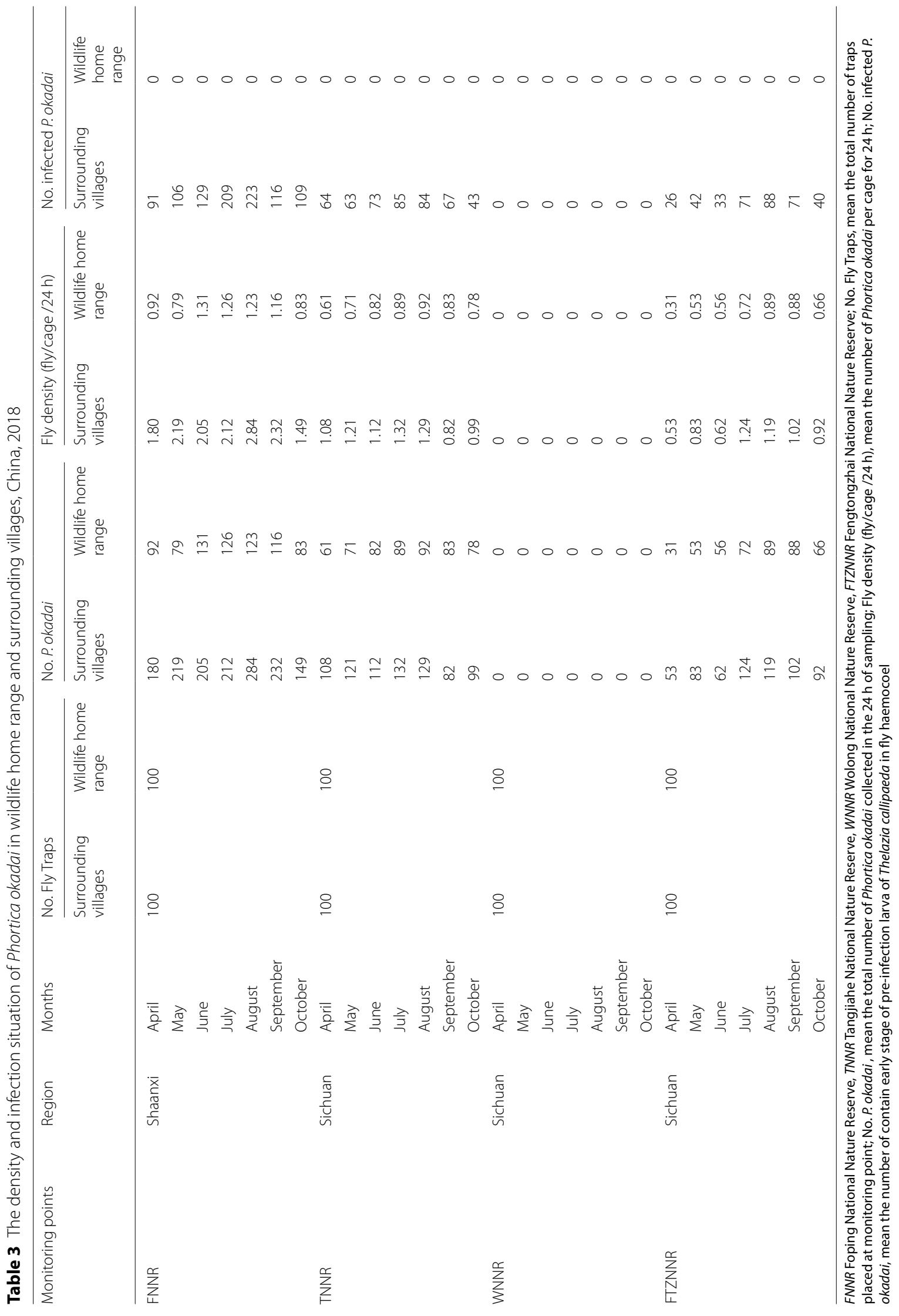




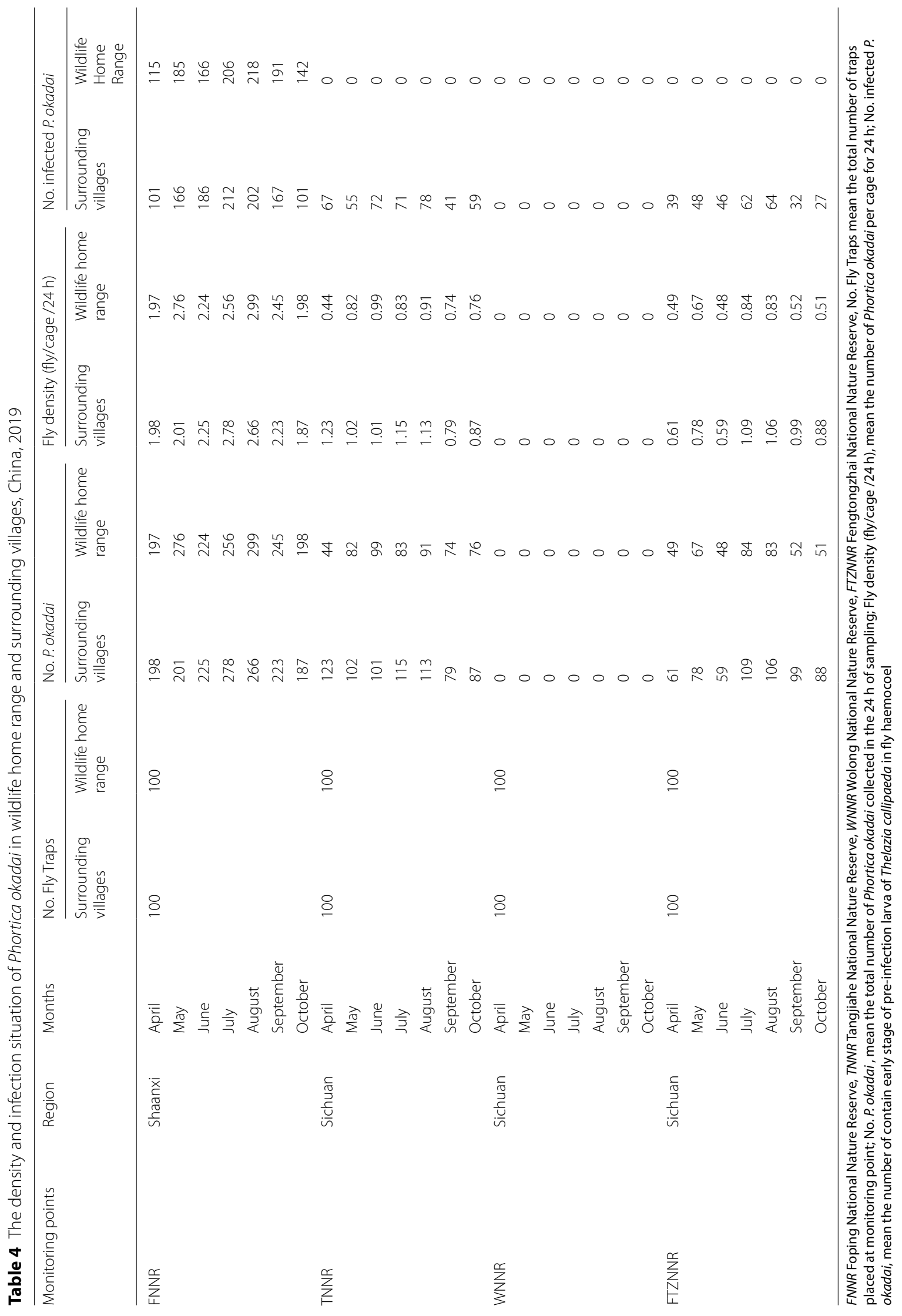



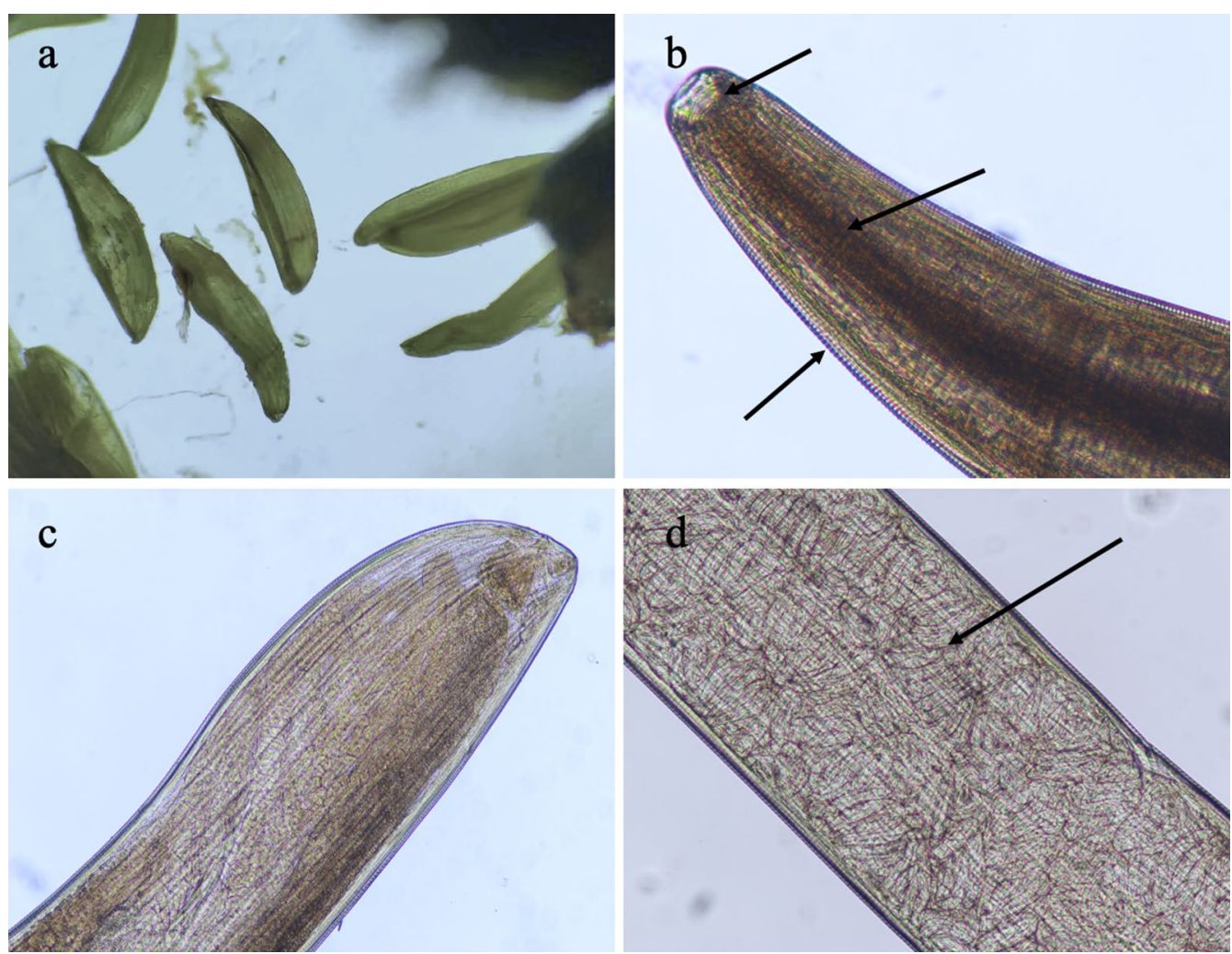

Fig. 3 a Early stage pre-infective larva of T. callipaeda found in the haemocoel of P. okadai. $\mathbf{b}$ Image of T. callipaeda showing the buccal capsule, pharynx, and esophagus of the anterior segment of the worm body and its serrated, wrinkled surface (arrow). c Caudal end of the worm. $\mathbf{d}$ Larvae in a female worm's uterus (arrow)

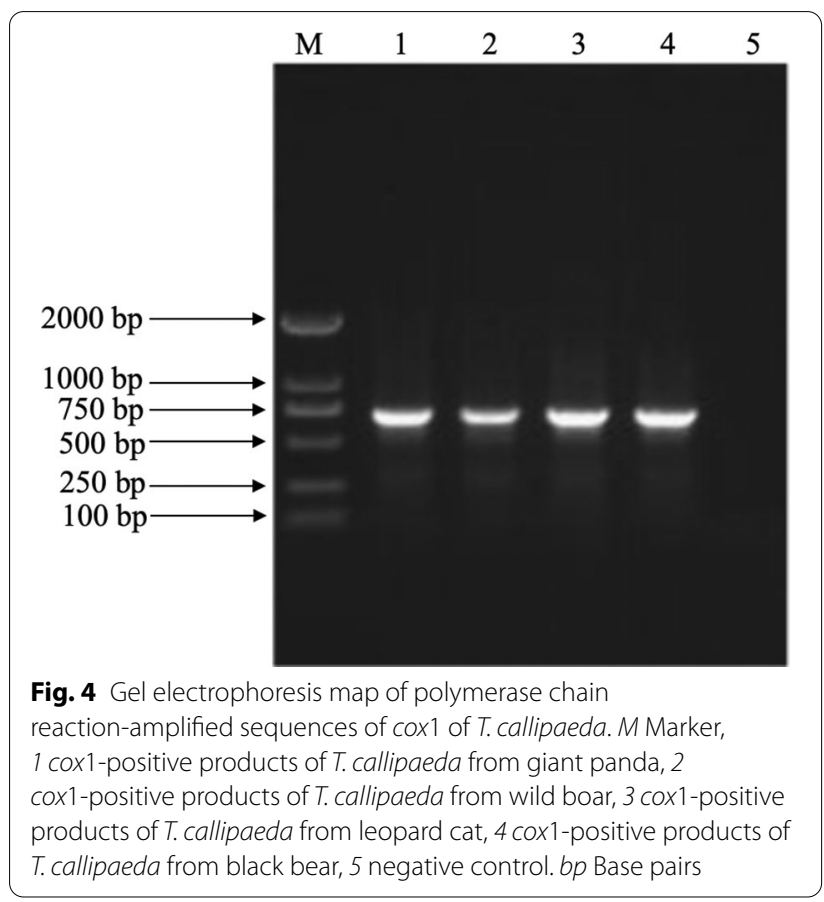

and mutation scanning the mitochondrial cyt1 [17]. Therefore, we believe that using cox 1 to reveal the genetic relationships and molecular evolution of T. callipaeda is extremely promising.

Although no cases of thelaziosis were found in wildlife in the three other reserves included in this study, we believe that continuous monitoring of the intermediate host needs to be carried out in these and in FNNR. In addition, domestic animals in the surrounding villages should be given deworming medicine regularly. These efforts would be helpful in monitoring the epidemiology of thelaziosis and weakening its threat to the public.

\section{Conclusions}

To the best of our knowledge, this is the first report of T. callipaeda infection in P. okadai, as well in a variety of wildlife, including giant panda, in nature reserves in China. The results of this study illustrate the important role of wildlife in vector-borne zoonoses. Further studies are needed to undertake risk assessments of T. callipaeda infection in the villagers who live around these nature reserves. 


\section{Supplementary Information}

The online version contains supplementary material available at https://doi. org/10.1186/s13071-020-04509-0.

Additional file 1: Figure S1. Thelazia callipaeda in the eye of a giant panda. Figure S2. Light micrographs of Phortica okadai. a White band around the compound eye (arrow); $\mathbf{b}$ multiple dark brown spots on the thorax (arrow); $\mathbf{c}$ three black bands on the tibia (arrow); $\mathbf{d}$ trident-shaped mark on the dorsal part of the abdomen (arrow)

\section{Acknowledgments}

This work was supported by a China Agricultural University Workstation located in FNNR. We gratefully acknowledge Xiaolin Wang, Yiwen He and Yidong He for their help with the data analysis, and Chang Yu and Qinghui Mu for their help with the preparation of the manuscript.

\section{Authors' contributions}

YPJ, ZCL, DGL, and JHL conceived the study. YPJ and ZCL organized the sampling plan. ZCL, JQW, YFW, NJH and LBT were responsible for the collection of cases of wild life thelaziosis used in this study. ZCL obtained the sequence of T. callipaeda and analyzed the results. All of the authors participated in the capture of P. okadai in the nature reserves. YPJ and ZCL produced the first draft of the manuscript, and all the authors contributed to the final version of the manuscript. All the authors read and approved the final manuscript.

\section{Funding}

Not applicable.

\section{Availability of data and materials}

The datasets supporting the conclusions of this article are included in the article. Sequences obtained during the current study are available from the GenBank database (accession numbers MN719908, MN719912, MN719913 and MN719914)

\section{Ethics approval and consent to participate}

Not applicable.

\section{Consent for publication}

Not applicable.

\section{Competing interests}

The authors declare that they have no competing interests.

\section{Author details}

${ }^{1}$ College of Veterinary Medicine, People's Republic of China Agricultural University, No. 2 Yuanmingyuan West Road, Haidian District, Beijing 100193, People's Republic of China. ${ }^{2}$ Foping National Nature Reserve, Shaanxi 723400, People's Republic of China.

Received: 26 July 2020 Accepted: 1 December 2020

Published online: 06 January 2021

\section{References}

1. Anderson RC. Nematode parasites of vertebrates: their development and transmission. 2nd ed. Wallingford: CABl; 2000

2. Máca J. Taxonomic notes on the genera previously classified in the genus Amiota Loew (Diptera: Drosophilidae, Steganinae). Acta Univ Carolinae Biol. 2003;43:247-74.

3. Otranto D, Lia RP, Buono V Traversa D. Biology of Thelazia callipaeda (Spirurida, Thelaziidae) eyeworms in naturally infected definitive hosts. Parasitology. 2004;129:627-33.

4. Otranto D, Lia RP, Cantacessi C, Testini G, Troccoli A, Shen JL, et al. The intermediate host of Thelazia callipaeda (Spirurida, Thelaziidae) in Europe and China: nematode biology and larval development inside the vector. Parasitology. 2005;131:847-55.

5. Wang ZX, Yang ZX. Thelaziasis definitive host and experimental anima studies investigating the host. Chin J Zoonoses. 1992;8:35-8.
6. Shen J Gasser R, Chu D, Wang Z, Yuan X, Cantacessi C, et al. Human thelaziosis - a neglected parasitic disease of the eye. J Parasitol. 2006:92:872-5.

7. Liao YL, Ren XH, Zheng MH, Wang LJ, Liu H. Epidemic characteristics of thelaziasis and species identification of Thelazia callipaeda by $18 \mathrm{~S}$ rRNA gene. Chin J Endemiol. 2017;36:557-61.

8. Malacrida F, Hegglin D, Bacciarini L, Otranto D, Nägeli F, Nägeli C, et al. Emergence of canine ocular thelaziosis caused by Thelazia callipaeda in southern Switzerland. Vet Parasitol. 2008;157:321-7.

9. Magnis J, Naucke TJ, Mathis A, Deplazes P, Schnyder M. Local transmission of the eye worm Thelazia callipaeda in southern Germany. Parasitol Res. 2010;106:715.

10. Ruytoor P, Déan E, Pennant O, Dorchies P, Chermette R, Otranto D, et al. Ocular thelaziosis in dogs in France. Emerg Infect Dis. 2010;16:1943-5.

11. Dorchies P, Chaudieu G, Siméon LA, Cazalot G, Cantacessi C, Otranto D. First reports of autochthonous eyeworm infection by Thelazia callipaeda (Spirurida, Thelaziidae) in dogs and cat from France. Vet Parasitol. 2007:149:294-7.

12. Ren $Y X$, Qin $Y H$, Zhang $L L$, Dai $X D$, Cui $Y$. A case report of human thelaziasis and its epidemiology in China. Chin J Zoo. 2017:33:1041-7.

13. Zhang X, Jiang P, Liu RD, Long SR, Cui J, Wang ZQ. Advances in etiology, epidemiology and genetic diversity of Thelazia callipaeda. Chin J Schisto Control. 2019;31:86-93.

14. Jin YP, Zhang XK, Ma YS, Qiao YC, Liu XB, Zhao KH, et al. Canine distemper viral infection threatens the giant panda population in China. Oncotarget. 2017:8:113910-9.

15. Leng PN, Zeng XP, Zhao TY, Xu RQ, Gao QF, Yu CJ. Surveillance methods for vector density-Fly. National Health Commission of the People's Republic of China. 2009. http://www.nhc.gov.cn/wjw/s9498/200908/ 42405.shtml. Accessed 4 May 2009.

16. Fuentes I, Montes I, Saugar MJ, Latrofa S, Gárate T, Otranto D. Thelaziosis in humans, a zoonotic infection, Spain, 2011. Emerg Infect Dis. 2012;18:2073-5.

17. Otranto D, Testini G, De Luca F, Hu M, Shamsi S, Gasser RB. Analysis of genetic variability within Thelazia callipaeda (Nematoda: Thelazioidea) from Europe and Asia by sequencing and mutation scanning of the mitochondrial cytochrome c oxidase subunit 1 gene. Mol Cell Probes. 2005:19:306-13.

18. Wang ZX, Wang KC, Chen Q. Experimental studies on susceptibility of Thelazia calipaeda to Amiota okadi in three provinces of China. Chin J Zoonoses. 2002;18:61-3.

19. Marino V, Gálvez R, Colella V, Sarquis J, Checa R, Montoya A, et al. Detection of Thelazia callipaeda in Phortica variegata and spread of canine thelaziosis to new areas in Spain. Parasites Vectors. 2018:11:195.

20. Otranto D, Ferroglio E, Lia RP, Traversa D, Rossi L. Current status and epidemiological observation of Thelazia callipaeda (Spirurida, Thelaziidae) in dogs, cats and foxes in Italy: a "coincidence" or a parasitic disease of the old continent? Vet Parasitol. 2003;116:315-25.

21. Maia C, Catarino AL, Almeida B, Ramos C, Campino L, Cardoso L. Emergence of Thelazia callipaeda infection in dogs and cats from east-centra Portugal. Transbound Emerg Dis. 2016:63:416-21.

22. Pombi M, Marino V, Jaenike J, Graham-Brown J, Bernardini I, Lia PR, et al. Temperature is a common climatic descriptor of lachryphagous activity period in Phortica variegata (Diptera: Drosophilidae) from multiple geographical locations. Parasites Vectors. 2020;13:89.

23. Hughes J, Macdonald DW. A review of the interactions between freeroaming domestic dogs and wildlife. Biol Conserv. 2013;157:341-51.

24. Loucks CJ, Lu Z, Dinerstein E, Wang DJ, Fu DL, Wang H. The giant pandas of the Qinling Mountains, China: a case study in designing conservation landscapes for elevational migrants. Conserv Biol. 2003;17:558-65.

25. Carter J, Ackleh AS, Leonard BP, Wang HB. Giant panda (Ailuropoda melanoleuca) population dynamics and bamboo (subfamily Bambusoideae) life history: a structured population approach to examining carrying capacity when the prey are semelparous. Ecol Model. 1999:123:207-23.

\section{Publisher's Note}

Springer Nature remains neutral with regard to jurisdictional claims in published maps and institutional affiliations. 